\title{
Angiotensin II promotes NO production, inhibits apopto- sis and enhances adhesion potential of bone marrow-de- rived endothelial progenitor cells
}

\author{
Tao Yin ${ }^{1}$, Xinliang $\mathrm{Ma}^{2}$, Li Zhao ${ }^{1}$, Kang Cheng ${ }^{1}$, Haichang Wang ${ }^{1}$ \\ ${ }^{1}$ Department of Cardiovascular Diseases, Xijing Hospital, Fourth Military Medical University, Xi'an, Shaanxi 710033, China; ${ }^{2}$ De- \\ partment of Emergency Medicine, Thomas Jefferson University, Philadelphia, PA 19107, USA
}

Endothelial progenitor cells (EPCs) participate in the processes of postnatal neovascularization and re-endothelialization in response to tissue ischemia and endothelial injury. The level of EPCs present has been found to be directly associated with the outcome of cardiovascular diseases, and could be regulated by stimulatory or inhibitory factors. Given the close relationship between angiotensin II (AngII) and the cardiovascular system, we investigated the effect of AngII on the activities of bone marrow (BM)-derived EPCs. Cells were isolated from BM of rats by density gradient centrifugation. Administration of AngII significantly promoted nitric oxide (NO) release, inhibited EPC apoptosis and enhanced EPC adhesion potential. All of these AngII-mediated effects on EPCs were attenuated by pretreatment with valsartan or L-NAME. Moreover, both LY294002 and wortmannin abolished the anti-apoptotic effect of AngII. Western blot analyses indicated that endothelial NO synthase (eNOS) protein and phosphorylated Akt increased with the treatment of AngII in EPCs. Thus, AngII improved several activities of EPCs through AngII type 1 receptor (AT1R), which may represent a possible mechanism linking AngII and AT1R with angiogenesis. Additionally, AngII-induced NO synthesis through eNOS in EPCs regulates apoptosis and adhesion, and the PI3-kinase/Akt pathway has an essential role in AngII-induced antiapoptosis signaling.

Keywords: endothelial progenitor cells, angiotensin, nitric oxide, apoptosis, adhesion

Cell Research (2008) 18:792-799. doi: 10.1038/cr.2008.69; published online 17 June 2008

\section{Introduction}

Endothelial progenitor cells (EPCs), also called angioblasts, have the capacity to differentiate into mature endothelial cells (ECs), and are considered to be precursors for ECs [1]. Accumulating evidence suggests that these precursors play a crucial role in the process of postnatal

Correspondence: Haichang Wang

Tel: +86-29-84773469; Fax: +86-29-84771170

E-mail: wanghc@fmmu.edu.cn

Abbreviations: endothelial progenitor cells (EPCs); endothelial cells (ECs); mononuclear cells (MNCs); angiotensin II (AngII); angiotensin II type 1 receptor (AT1R); renin-angiotensin system (RAS); nitric oxide (NO); bone marrow (BM); peripheral blood (PB); vascular endothelial growth factor (VEGF); basic fibroblast growth factor (bFGF); vascular endothelial growth factor receptor-2 (VEGFR-2); 3-(4,5-dimethylthiazol-2-yl)-2,5-diphenyltetrazolium bromide (MTT); endothelial nitric oxide synthase (eNOS); angiotensin converting enzyme inhibitor (ACEI); angiotensin receptor blockers (ARB) Received 29 March 2007; revised 18 October 2007; accepted 14 January 2008; published online 17 June 2008 neovascularization, which is a vital compensatory response to tissue ischemia, and contribute to re-endothelialization after endothelial injury [2].

However, the number and function of EPCs have been shown to inversely correlate with risk factors for cardiovascular disease [3, 4]. Numerous clinical and experimental studies have demonstrated that angiotensin II (AngII) is crucial in the development of cardiovascular diseases, but it remains unclear whether AngII regulates the activities of EPCs. On the other hand, increasing evidence has demonstrated the role of AngII in angiogenesis [5], suggesting that AngII may improve the activities of EPCs.

AngII inhibits EC apoptosis [6], upregulates adhesion molecules [7] and potently augments endothelial nitric oxide synthase (eNOS) mRNA expression, which thereby increases nitric oxide (NO) release [8]. Therefore, we propose that AngII may have similar effects on EPCs as well. In the present study, we examined the effects of different concentrations of AngII on NO release, eNOS protein expression, apoptosis and adhesion potential in isolated 
EPCs. We also evaluated the role of AngII type 1 receptor (AT1R) in these processes and the crosstalk between AngIIinduced NO synthesis and apoptosis or adhesion by using L-NAME, a NOS inhibitor. Furthermore, we investigated the contribution of the PI3-kinase/Akt pathway to AngIIinduced antiapoptosis signaling.

\section{Results}

Cells in the present study demonstrated the characteristics of EPCs

After 7 days of culture, EPCs were visualized by inverted microscopy and characterized by fluorescence staining. Ex vivo expanded EPCs derived from bone marrow (BM) of Sprague Dawley (SD) rats exhibited a spindle-shaped morphology [1] (Figure 1A). The stem/progenitor cell qualities of the EPCs were evidenced by their proliferative capacity to form endothelial colonies [1, 4] (Figure 1B). EPCs were qualified as adherent cells stained double positive for AC133 and vascular endothelial growth fac- tor receptor-2 (VEGFR-2/Flk-1) using a laser scanning confocal microscope (Figure 1C), corresponding to the previously described phenotype $[2,9]$.

\section{EPCs expressed AT1R}

RT-PCR was performed to determine the presence of AT1R in EPCs using total RNA derived from cells after 7 days of culture. RT-PCR analysis indicated that AT1R mRNA exists in EPCs (Figure 2). Therefore, AngII might have an effect on EPCs.

AngII increased NO secretion and expression of eNOS protein in EPCs

NO levels in media obtained from EPCs treated with AngII or vehicle were measured using an NO assay kit. AngII caused elevated NO secretion in EPCs, and this stimulatory effect reached statistical significance at all doses studied (Figure 3A). Valsartan, an AT1R antagonist, and L-NAME, a NOS inhibitor, blocked the stimulatory effect of AngII (Figure 3B).
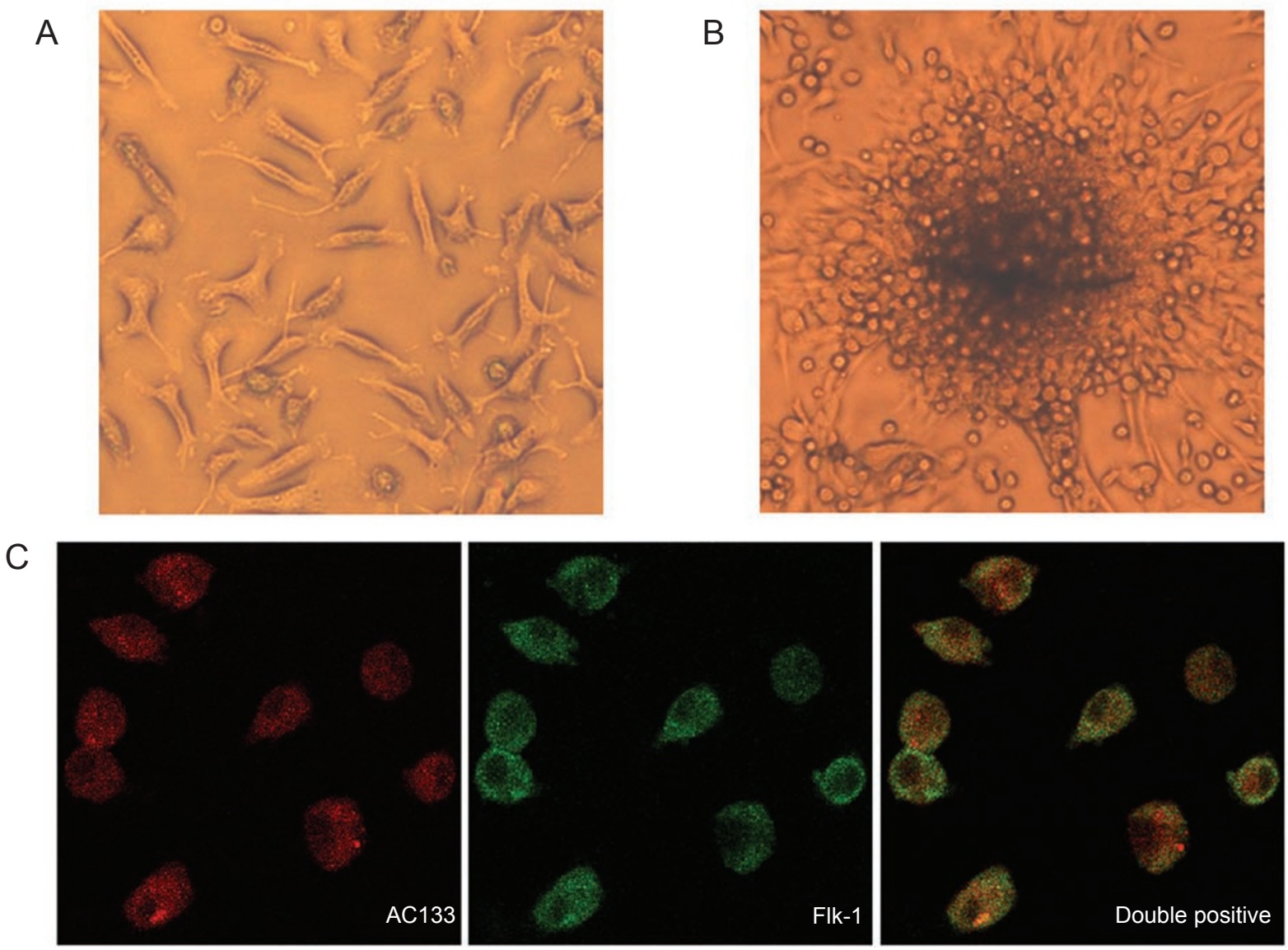

Figure 1 (A) Attached cells exhibit a spindle-shaped, endothelial cell-like morphology $(\times 100)$. (B) EPCs show their proliferative capacity to form endothelial colonies (×100). (C) Expression of AC133 (red) and Flk-1 (green) was assessed under LSCM. Double-positive cells (yellow) were identified as differentiating EPCs $(\times 200)$. 


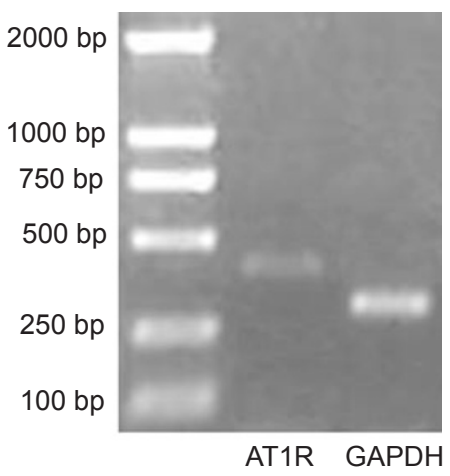

Figure 2 Expression of AT1R in EPCs as shown by RT-PCR. To confirm the presence of AT1R in EPCs, RT-PCR was performed. The expected sizes of the RT-PCR products were $430 \mathrm{bp}$ (AT1R) and $307 \mathrm{bp}$ (GAPDH).

We investigated the expression of eNOS protein in EPCs using western blot analysis. It was shown that EPCs expressed eNOS protein, and the levels of eNOS increased under AngII stimulation (Figure 4). Our data indicate that AngII may potentiate NO release by increasing the expression of eNOS.

AngII exerted antiapoptotic effect in EPCs through PI3kinase/Akt pathway

To examine the effect of AngII on the survival of ex vivo expanded EPCs, we measured the apoptosis that was induced by serum starvation. Hoechst staining was performed to determine the proportion of apoptotic cells by manually counting pyknotic nuclei. Typical apoptosis led to the cells shrinking and the nuclei became condensed (Figure 5A). Approximately $26 \%$ of EPCs underwent apoptosis after $24 \mathrm{~h}$ of serum deprivation, and AngII reduced apoptosis in EPCs (Figure 5B). Valsartan suppressed the antiapoptotic effect of AngII (Figure 5C).

We also investigated the signaling molecule PI3-kinase/Akt, which lies downstream of AT1R. LY294002 and wortmannin, two kinds of PI3-kinase/Akt inhibitors, both abolished the antiapoptotic effect of AngII (Figure 5C). Western blot analysis indicated that incubation with AngII significantly increased phosphorylated Akt (Ser473) levels in EPCs (Figure 4). Therefore, this suggests that AngII exerts its antiapoptotic effect in EPCs through the PI3-kinase/Akt pathway.

AngII enhanced potential of adhesion to matrix in EPCS

Crystal violet staining demonstrated that treatment with AngII increased the number of EPCs attached to fibronectin-coated culture dishes, with a maximal effect
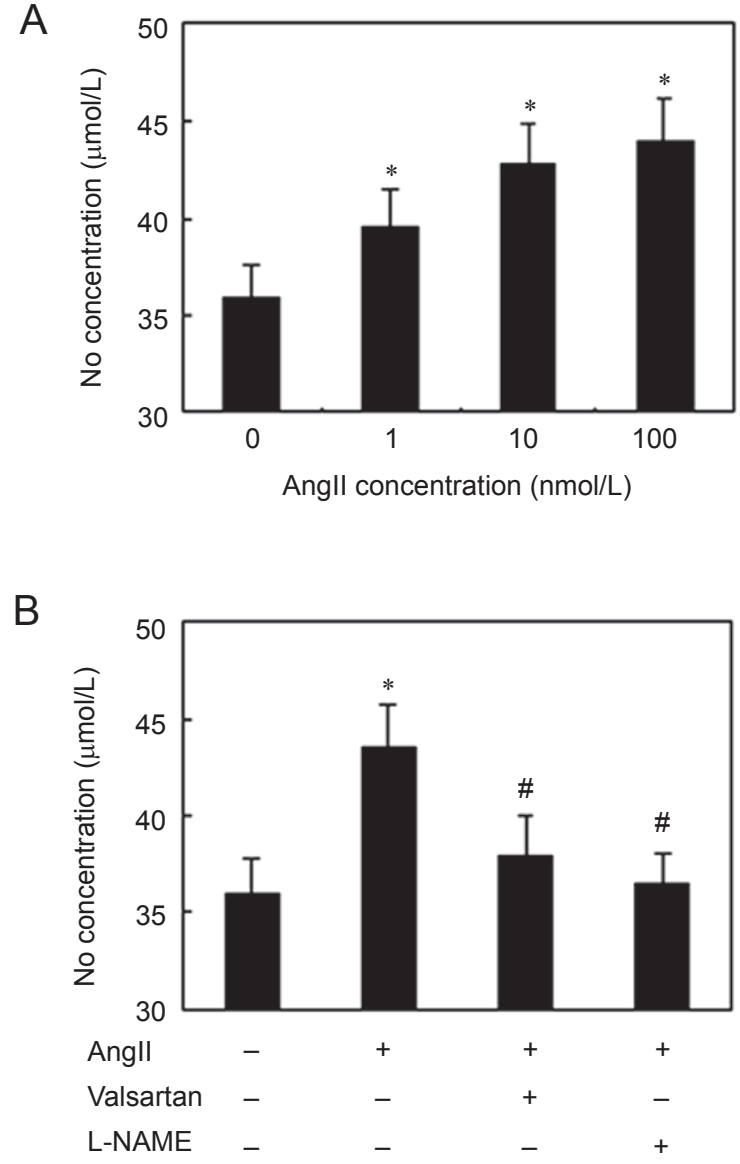

Figure 3 Effect of Angll on NO release and expression of eNOS in EPCs. (A) Dose-response analysis of the stimulatory effect of Angll on NO secretion in EPCs. Cells were treated with either vehicle or Ang II (1 to $100 \mathrm{nmol} / \mathrm{L}$ ) for $24 \mathrm{~h}$. NO concentrations in supernatants from cultured EPCs were measured using a NO assay kit. (B) Role of AT1R and eNOS in the stimulatory effect of Angll. Cells were pretreated for $30 \mathrm{~min}$ with vehicle, valsartan $(1 \mu \mathrm{mol} / \mathrm{L})$, or L-NAME $(100 \mu \mathrm{mol} / \mathrm{L})$, and then incubated with Ang II (100 nmol/L) for $24 \mathrm{~h} . * P<0.05$ versus control, ${ }^{*} P<0.05$ versus Angll.

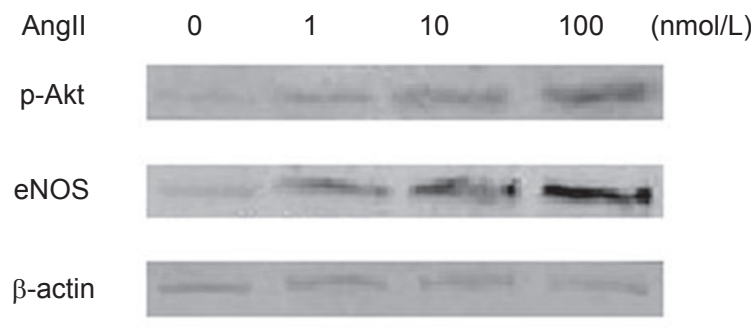

Figure 4 Effect of Angll on phosphorylation of Akt and expression of eNOS protein in EPCs. Cells were treated with either vehicle or Ang II (1-100 nmol/L) for $24 \mathrm{~h}$, and then western blot analysis was performed. Representative blots were shown. 
A

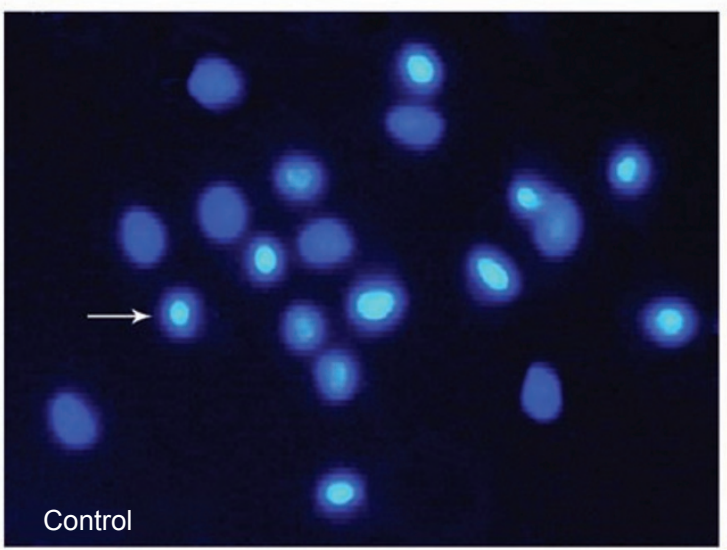

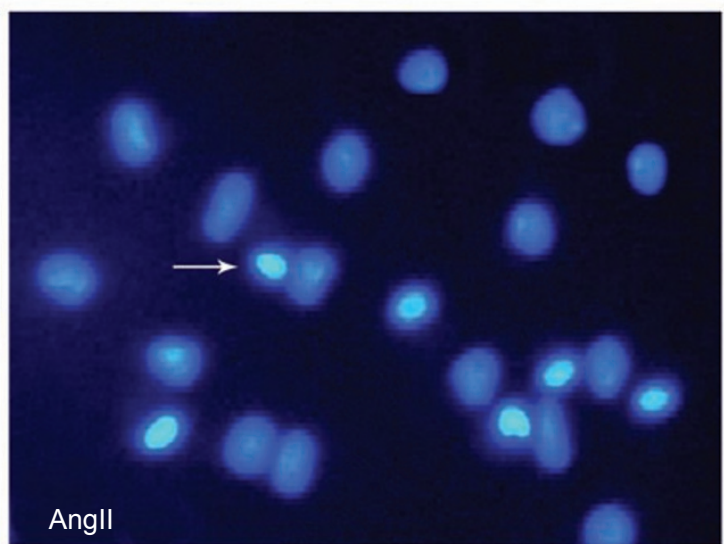

Angll
B

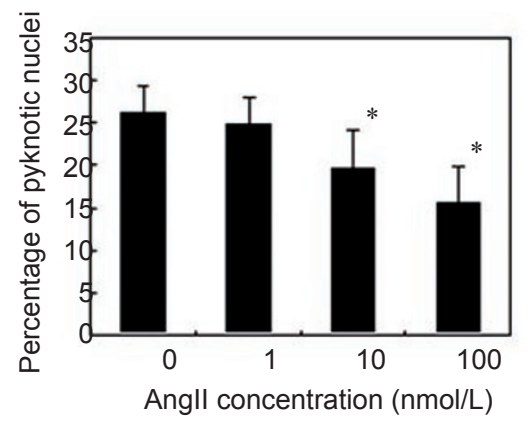

C

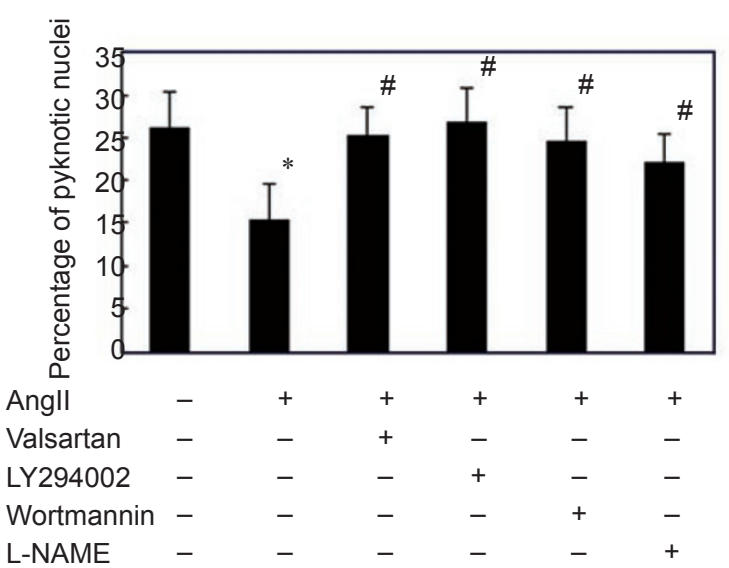

Figure 5 Effect of Angll on apoptosis in EPCs. Serum starvation was used to induce apoptosis in ex vivo expanded EPCs. (A) Hoechst staining was performed to determine the ratio of apoptotic cells by manually counting white condensed pyknotic nuclei (arrows point to the representative apoptotic nuclei in figures, $\times 200$ ). (B) Dose-response analysis of the antiapoptotic effects of Angll on EPCs. Cells were treated with either vehicle or Ang II (1-100 nmol/L) for $24 \mathrm{~h}$ after changing to serum-free medium. (C) Role of AT1R, the PI3-kinase/Akt pathway and NO in Angll-induced antiapoptosis. Cells were pretreated for 30 min with vehicle, valsartan $(1 \mu \mathrm{mol} / \mathrm{L})$, LY294002 (100 $\mu \mathrm{mol} / \mathrm{L})$, wortmannin $(100 \mathrm{nmol} / \mathrm{L})$, or L-NAME (100 $\mu \mathrm{mol} / \mathrm{L})$, and then incubated with Ang II $(100 \mathrm{nmol} / \mathrm{L})$ for $24 \mathrm{~h}$ in serum-free medium. ${ }^{*} P<0.05$ versus serum-deprived control, ${ }^{*} P<0.05$ versus Angll.

achieved at $100 \mathrm{nmol} / \mathrm{L}$ (Figure 6A). This result indicates that AngII enhances the potential of adhesion to matrix in a dose-dependent manner in EPCs. The effect appears to be mediated through AT1R as it is blocked by valsartan (Figure 6B).

AngII exerted antiapoptotic and adhesion-promoting effects in EPCs partly through increased NO synthesis

To study the functional effects of AngII-induced NO synthesis, we also used L-NAME in apoptosis and adhesion assays. The results indicate that L-NAME attenuated the antiapoptotic (Figure 5C) and adhesion-promoting effects of AngII (Figure 6B). Thus, it is suggested that AngII-induced NO synthesis may play a role in improving the other two activities of EPCs as well.

AngII, in the absence of vascular endothelial growth factor (VEGF), had no effect on proliferation activity in EPCs

In the absence of VEGF, AngII had no effect on the proliferation activity of EPCs (Figure 7), which is consistent with a previous study by Imanishi et al. [10]. This result excludes a spurious effect of stimulated proliferation on the evaluation of other AngII stimulatory effects on EPCs.

\section{Discussion}

Previous studies have identified a population of cells, termed EPCs, that were isolated from peripheral blood 
A

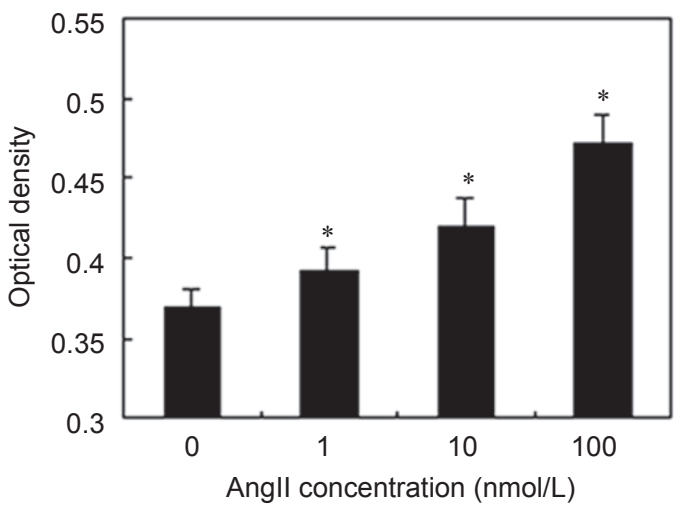

B

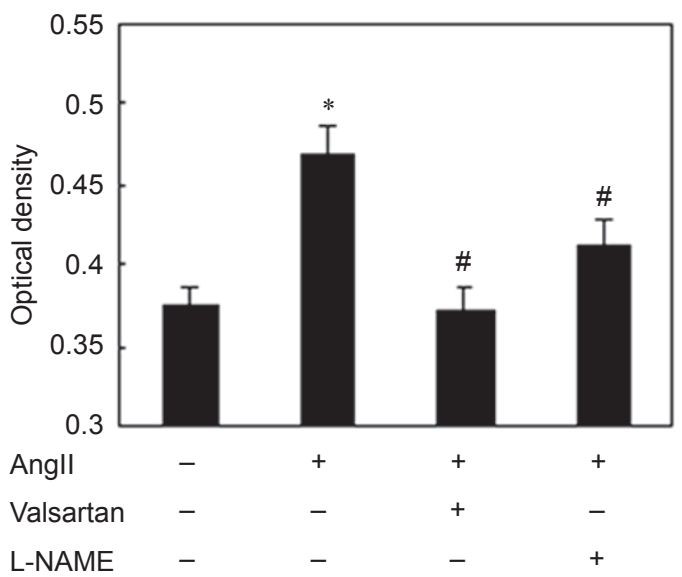

Figure 6 Effect of Angll on adhesion in EPCs. Attached cells were quantified by analyzing the optical density after staining with crystal violet. (A) Dose-response analysis of the effect of Angll on adhesion potential in EPCs. Cells were treated with either vehicle or Ang II (1-100 nmol/L) for $24 \mathrm{~h}$. (B) Role of AT1R and NO in the effect of Angll. Cells were pretreated with vehicle, valsartan (1 $\mu \mathrm{mol} / \mathrm{L})$ or L-NAME $(100 \mu \mathrm{mol} / \mathrm{L})$ for $30 \mathrm{~min}$, and then incubated with Ang II (100 nmol/L) for $24 \mathrm{~h} . * P<0.05$ versus control, ${ }^{*} P<$ 0.05 versus Angll.

(PB) $[1,3,9]$, BM $[11,12]$ and umbilical cord blood mononuclear cells (MNCs) [13]. These cells differentiate into ECs in vitro and in vivo, and incorporate into sites of active angiogenesis $[1,2]$. Laboratory evidence has indicated that EPCs participate not only in the process of vasculogenesis, the term applied to the formation of embryonic blood vessels, but also in the process of postnatal neovascularization and repair of injured endothelium [2, 14, 15]. EPCs can be mobilized and aggregate to the foci of vascular injury or ischemia, affecting vascular repair capacity and local perfusion. CD133, also known as prominin or AC133, is expressed on hematopoietic stem/progenitor cells but is absent on mature ECs, and VEGFR-2 is a kind of endothelial marker protein $[2,9]$. Thus, cells co-expressing CD133 and
VEGFR-2 in our study may represent EPCs.

AngII, an octapeptide hormone, is an important active element in the renin-angiotensin system (RAS). It not only has significant physiological influence on the regulation of systemic hemodynamics and plasma volume, but also plays a pathophysiological role in hypertension, atherosclerosis and cardiac remodeling through its hypertrophic and proliferative growth actions. Nevertheless, the role of AngII in angiogenesis has only recently begun to be evaluated [5]. Siddiqui et al. [16] observed that enalapril and candesartan both specifically inhibit phVEGF-A165-induced myocardial angiogenesis in the normal heart. Michel and colleagues [17] also reported that aldosterone increases neovascularization in the setting of ischemia through activation of AngII signaling. Despite these findings, the mechanism by which AngII promotes angiogenesis is still elusive. In the present study, we demonstrated that AngII has several favorable effects on EPCs, which are important in angiogenesis, that may shed light on the mechanism.

Imanishi et al. [10] have previously reported that AngII potentiates VEGF-induced EPC proliferation and network formation. However, the effect of AngII on the other activities of EPCs remains unclear. Moreover, in their study, EPCs were isolated from PB-MNCs. PB-MNCs effectively improve collateral perfusion primarily through their ability to supply angiogenic factors and cytokines. The implanted PB-MNCs were not incorporated into neocapillaries, whereas the implanted BM-MNCs were shown to differentiate into vascular ECs $[18,19]$. In addition, it has been shown that re-endothelialization at sites of vascular damage is also modulated by the mobilization and incorporation of BM-derived EPCs [20]. Therefore, we chose to investigate

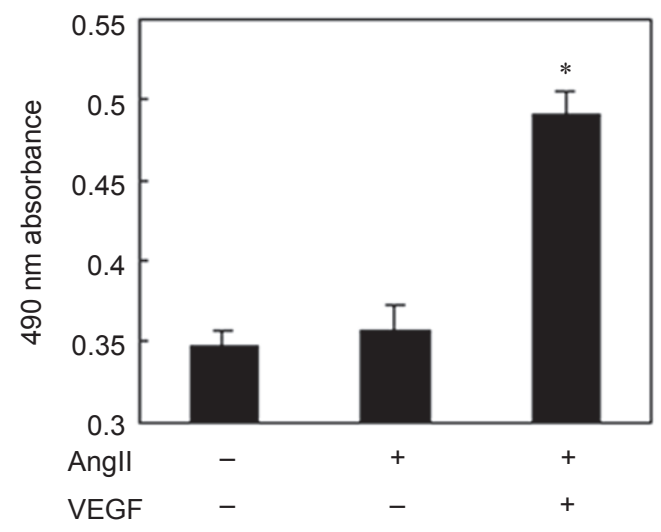

Figure 7 Effect of Angll on proliferation in EPCs. Cells were pretreated with either vehicle or Angll (100 nmol/L) for $12 \mathrm{~h}$, and then vehicle or VEGF $(20 \mathrm{ng} / \mathrm{ml})$ was added into the medium. After treatment for $24 \mathrm{~h}$, MTT assay was performed. $* P<0.05$ versus control. 
the relationship between AngII and BM-EPCs.

We observed the effects of AngII on several activities of EPCs as follows. First, AngII increased eNOS expression and thus NO release. Mobilization and differentiation of EPCs is known to be modified by NO [21]. Observations that mice deficient in eNOS display reduced EPCs mobilization by VEGF and impaired ischemia-induced angiogenesis [22] suggest that eNOS may be required for EPC-induced angiogenesis. Thus, AngII-mediated upregulation of eNOS and NO in EPCs may be responsible for its favorable effects on neovascularization. Second, AngII inhibits apoptosis of EPCs under serum starvation conditions, which may help maintain the number of EPCs when they are recruited to ischemic regions. We further investigated the mechanisms underlying this effect by examining the PI3-kinase/Akt pathway, which lies downstream of AT1R [6]. It was found that the pathway also plays an essential role in the AngII-induced antiapoptotic signaling of EPCs. Moreover, we found that NO mediates the antiapoptotic effect of AngII as well. This provides further evidence that NO acts as a survival signal for cells. Third, AngII enhanced EPC adhesion capacity. Adhesion to the extracellular matrix is thought to be important during new vessel growth for EPCs [2, 15]. Enhanced adhesion of EPCs to matrix under AngII stimulation may determine its homing to and incorporation into regions of ischemic or vascular injury. Lee et al. [23] reported that NO upregulates integrin, which mediates cell-matrix adhesion. We observed that LNAME attenuates the adhesion-promoting effects of AngII on EPCs. This provides further support to the idea that NO modulates cell-matrix adhesion. Finally, the AT1R agonist valsartan suppresses the favorable effects of AngII on the biology of EPCs. This indicates that AngII may improve the activity of EPCs through AT1R. Sasaki et al. [24] reported that the formation of well-developed collateral vessels in response to hindlimb ischemia was reduced in AT1aR knockout mice, and that treatment of wild-type mice with the AT1R blocker also impairs ischemia-induced angiogenesis. Toko et al. [25] showed that neovascularization after myocardial infarction is less prominent in ATlaR-deficient mice. These results, combined with ours, suggest that AT1R has an important role in promoting early angiogenesis.

From a clinical point of view, despite accumulating evidence to show that cardiovascular diseases such as atherosclerosis, restenosis after vascular interventions and myocardial regeneration after infarction are influenced by EPCs $[15,26,27]$, the factors that affect the number and function of EPCs have just begun to be identified. Several cardiovascular risk factors have been shown to be associated with dysfunction and decreased numbers of EPCs, such as hypercholesterolemia [28], diabetes [29] and Creactive protein [27]. Conversely, exercise [30], statins [28] and estrogen [31] positively regulate EPCs. Additionally, in our study, AngII was also shown to be such a positive factor. As a result, the balance between these two kinds of factors may influence EPC levels and thereby the capacity for neovascularization and re-endothelialization.

It is well known that AngII is an important risk factor related to cardiovascular disease. Drugs targeting RAS have been widely applied in the treatment of cardiovascular disease. Therefore, this points to a need for a better understanding of the relationship between AngII and EPCs. The present study was designed to investigate the biological effects of AngII on EPCs. Interestingly, we found that AngII, a cardiovascular risk factor, could improve the activity of EPCs, which seems to be contradictory to the clinical application in which two kinds of favorable drugs are used for patients with cardiovascular diseases: angiotensin converting enzyme inhibitor (ACEI) and angiotensin receptor blocker (ARB). It is likely that there are other mechanisms by which ACEI and ARB exert their beneficial effects. If so, it may be necessary to determine an optimal time window for the administration of ACEI and ARB in future.

In conclusion, the present study has revealed novel beneficial effects of AngII on the activities of BM-EPCs. A better understanding of the actions of EPCs will enable the design of new pharmacological therapies or regeneration strategies related to EPCs.

\section{Materials and Methods}

\section{Animals}

SD rats at the age of 4 weeks, weighing 80-100 g, were purchased from the Experimental Animal Center of the Fourth Military Medical University (Xi'an, China). All experiments were conducted in conformity with the Institutional Guidelines for the Care and Use of Laboratory Animals.

\section{Isolation and cultivation of EPCs}

EPCs were cultured according to the techniques described previously [11]. Briefly, hollow femurs and tibias of SD rats were prepared using standard surgical procedures and whole BM was harvested by flushing the marrow with Medium 199 (M199, Gibco) using a sterilized syringe. Total MNCs were isolated from a cell suspension containing BM through Ficoll density gradient centrifugation, and then washed twice. Isolated cells were subsequently plated onto human fibronectin (Chemicon) coated dishes at a density of $1 \times$ $10^{6} \mathrm{MNCs} / \mathrm{cm}^{2}$ in M199 supplemented with $10 \%$ fetal-calf serum, recombinant rat VEGF (10 ng/ml, R\&D Systems), recombinant human basic fibroblast growth factor (bFGF) $(10 \mathrm{ng} / \mathrm{ml}$, TBD Science), penicillin $(100 \mathrm{U} / \mathrm{ml})$ and streptomycin $(100 \mu \mathrm{g} / \mathrm{ml})$. On day 4 , adherent cells were washed extensively to remove unattached cells, fresh growth media was added and the culture was maintained for 7 days. All experiments below were performed using first passage cells. Day-7 EPCs were lifted with trypsinization, re-suspended in M199 supplemented with 10\% fetal-calf serum and then re-plated onto a culture plate for $24 \mathrm{~h}$ before treatment with reagents. 


\section{Cellular characterization}

The cell morphology was viewed with an inverted microscope (Nikon). Immunohistochemistry was used to determine the EPC phenotype after $7 \mathrm{~d}$ in culture. EPCs were fixed in 4\% paraformaldehyde for 30 min, washed 3 times with PBS, and dual-stained with goat polyclonal antibody against AC133 (Santa Cruz) and mouse polyclonal antibody against VEGFR-2 (Flk-1) (Santa Cruz). The presence of antibody was confirmed by exposure of the cells to TRITC-conjugated anti-goat secondary antibody and FITC-conjugated anti-mouse secondary antibody (Zymed). Cells were then visualized under a laser scanning confocal microscope (LSCM, Leica) and those cells demonstrating double-positive fluorescence were identified as EPCs.

\section{Expression of AT1R in EPCs}

Expression of AT1R in EPCs was detected by reverse transcription-polymerase chain reaction (RT-PCR). Total RNA was isolated from EPCs using an RNA extraction kit (Promega) and cDNA was synthesized by a reverse transcription kit (Promega) according to the manufacturer's instructions. PCR was performed using the following primers obtained from Genebank: AT1R, forward: 5'-AGC TGT CAT CCA CCG AAA TG-3', reverse: 5'-CAG AAA GCC GTA GAA CAG AG-3'; GAPDH, forward: 5'-TGAACG GGAAGC TCA CTG GC-3', reverse: 5'-TCC ACC ACC CTG TTG CTG TA-3'.

Reaction conditions were set as follows: $94{ }^{\circ} \mathrm{C}(2 \mathrm{~min}), 94{ }^{\circ} \mathrm{C}(45$ s), $55^{\circ} \mathrm{C}(45 \mathrm{~s})$ and $72{ }^{\circ} \mathrm{C}(45 \mathrm{~s})$ for 30 cycles and $5 \mathrm{~min}$ at $72{ }^{\circ} \mathrm{C}$. PCR products were fractionated by $2 \%$ agarose gel electrophoresis, stained with ethidium bromide, visualized under ultraviolet light and then photographed.

\section{NO assay}

The amount of NO released by EPCs was determined using an NO assay kit (Nanjing Jiancheng Institute of Biological Engineering, China) according to the manufacturer's protocol. Briefly, $100 \mu 1$ supernatants from cultured EPCs were harvested after the different stimulations, and supernatant $\mathrm{NO}$ levels were determined by colorimetric assay. The absorbance was measured spectrophotometrically at $550 \mathrm{~nm}$ after enzymatic conversion of the supernatant nitrate to nitrite by nitrate reductase.

\section{Apoptosis assay}

Apoptosis of EPCs, induced by serum starvation as described [32], was quantified to determine whether AngII exerts a survival effect on EPCs. The proportion of apoptotic EPCs after serum starvation was determined by manually counting pyknotic nuclei after Hoechst33258 (Sigma) staining. In brief, cells in culture plates were treated with different reagents after changing to serum-free medium and the ratio of apoptotic cells was determined after $24 \mathrm{~h}$. Each group was studied at least in triplicate.

\section{Adhesion assay}

An adhesion activity assay of EPCs was performed by staining with crystal violet as described [29]. After treatment with different reagents, the EPCs were washed with PBS and gently detached with $0.25 \%$ trypsin. Identical numbers of cells were re-plated onto fibronectin-coated culture dishes and incubated for $30 \mathrm{~min}$ at $37^{\circ} \mathrm{C}$. Adherent cells were stained with $0.1 \%$ crystal violet and rinsed with $10 \%$ acetic acid to elute the stain from the cells. Attached cells were quantified by analyzing the optical density of the media at a wavelength of $600 \mathrm{~nm}$ with a microtiter plate reader.

\section{Western blot analysis}

After stimulation with different reagents, cells were washed three times in PBS, and then scraped using an ice-cold lysis buffer. Total proteins obtained by centrifugation were separated on $8 \%$ SDS-PAGE gels and transferred electrophoretically to nitrocellulose membranes. After blocking with 5\% skim milk, the membranes were incubated with rabbit polyclonal antibodies against eNOS and phosphorylated Akt Ser473 (Cell Signaling Technology) at $4{ }^{\circ} \mathrm{C}$ overnight. The membranes were then washed and further incubated with the secondary antibody (peroxidase-labeled anti-rabbit IgG, Cell Signaling Technology) at $37{ }^{\circ} \mathrm{C}$ for $60 \mathrm{~min}$. Blots were visualized using an enhanced chemiluminescence system (ECL; Amersham).

\section{Proliferation assay}

To avoid bias attributable to AngII-induced EPC proliferation in the parameters measured above, AngII-induced EPC proliferation was assessed by 3-(4,5-dimethylthiazol-2-yl)-2,5-diphenyltetrazolium bromide (MTT) assay as described [10]. Although Imanishi et al. [10] have reported the relationship between AngII and EPCs, we investigated this effect of AngII again because of the different origin of EPCs in our study. After incubation with different reagents, cells were treated with MTT ( $5 \mathrm{~g} / \mathrm{l}$, Sigma) for $4 \mathrm{~h}$ at $37^{\circ} \mathrm{C}$. The cell culture medium was then removed, cells were lysed in $200 \mu$ l dimethyl sulfoxide (DMSO), and the amount of MTT was photometrically determined at a wavelength of $490 \mathrm{~nm}$.

\section{Statistical analysis}

The results are presented as the mean $\pm \mathrm{SD}$. Statistical analysis was performed by ANOVA for multiple comparisons, followed by LSD- $t$ correction for the post-hoc $t$ test. Differences were considered statistically significant when $P<0.05$. All analyses were performed with SPSS 11.0.

\section{Acknowledgments}

This work was supported by the National Natural Science Foundation of China (No. 30370581).

\section{References}

1 Asahara T, Murohara T, Sullivan A, et al. Isolation of putative progenitor endothelial cells for angiogenesis. Science 1997; 275:964-967.

2 Urbich C, Dimmeler S. Endothelial progenitor cells: characterization and role in vascular biology. Circ Res 2004; 95:343-353.

3 Vasa M, Fichtlscherer S, Aicher A, et al. Number and migratory activity of circulating endothelial progenitor cells inversely correlate with risk factors for coronary artery disease. Circ Res 2001; 89:E1-E7.

4 Hill JM, Zalos G, Halcox JP, et al. Circulating endothelial progenitor cells, vascular function, and cardiovascular risk. $N$ Engl $J$ Med 2003; 348:593-600.

5 Ichiki T. Role of renin angiotensin system in angiogenesis: it is still elusive. Arterioscler Thromb Vasc Biol 2004; 24:622-624.

6 Ohashi H, Takagi H, Oh H, et al. Phosphatidylinositol 3-kinase/ Akt regulates angiotensin II-induced inhibition of apoptosis in 
microvascular endothelial cells by governing survivin expression and suppression of caspase-3 activity. Circ Res 2004; 94:785793.

7 Pastore L, Tessitore A, Martinotti S, et al. Angiotensin II stimulates intercellular adhesion molecule-1 (ICAM-1) expression by human vascular endothelial cells and increases soluble ICAM-1 release in vivo. Circulation 1999; 100:1646-1652.

8 Zheng J, Bird IM, Chen DB, Magness RR. Angiotensin II regulation of ovine fetoplacental artery endothelial functions: interactions with nitric oxide. J Physiol 2005; 565:59-69.

9 Peichev M, Naiyer AJ, Pereira D, et al. Expression of VEGFR2 and $\mathrm{AC} 133$ by circulating human CD34 $(+)$ cells identifies a population of functional endothelial precursors. Blood 2000; 95:952-958.

10 Imanishi T, Hano T, Nishio I. Angiotensin II potentiates vascular endothelial growth factor-induced proliferation and network formation of endothelial progenitor cells. Hypertens Res 2004; 27:101-108.

11 Thum T, Fraccarollo D, Galuppo P, et al. Bone marrow molecular alterations after myocardial infarction: impact on endothelial progenitor cells. Cardiovasc Res 2006; 70:50-60.

12 Reyes M, Dudek A, Jahagirdar B, Koodie L, Marker PH, Verfaillie CM. Origin of endothelial progenitors in human postnatal bone marrow. J Clin Invest 2002; 109:337-346.

13 Murohara T, Ikeda H, Duan J, et al. Transplanted cord bloodderived endothelial precursor cells augment postnatal neovascularization. J Clin Invest 2000; 105:1527-1536.

14 Carmeliet P. Mechanisms of angiogenesis and arteriogenesis. Nat Med 2000; 6:389-395.

15 Szmitko PE, Fedak PW, Weisel RD, Stewart DJ, Kutryk MJ, Verma S. Endothelial progenitor cells: new hope for a broken heart. Circulation 2003; 107:3093-3100.

16 Siddiqui AJ, Mansson-Broberg A, Gustafsson T, et al. Antagonism of the renin-angiotensin system can counteract cardiac angiogenic vascular endothelial growth factor gene therapy and myocardial angiogenesis in the normal heart. Am J Hypertens 2005; 18:1347-1352.

17 Michel F, Ambroisine ML, Duriez M, Delcayre C, Levy BI, Silvestre JS. Aldosterone enhances ischemia-induced neovascularization through angiotensin II-dependent pathway. Circulation 2004; 109:1933-1937.

18 Asahara T, Masuda H, Takahashi T, et al. Bone marrow origin of endothelial progenitor cells responsible for postnatal vasculogenesis in physiological and pathological neovas-cularization. Circ Res 1999; 85:221-228.

19 Kamihata H, Matsubara H, Nishiue T, et al. Improvement of collateral perfusion and regional function by implantation of peripheral blood mononuclear cells into ischemic hibernating myocardium. Arterioscler Thromb Vasc Biol 2002; 22:18041810.
20 Werner N, Priller J, Laufs U, et al. Bone marrow-derived progenitor cells modulate vascular reendothelialization and neointimal formation: effect of 3-hydroxy-3-methylglutaryl coenzyme a reductase inhibition. Arterioscler Thromb Vasc Biol 2002; 22:1567-1572.

21 Thum T, Tsikas D, Stein S, et al. Suppression of endothelial progenitor cells in human coronary artery disease by the endogenous nitric oxide synthase inhibitor asymmetric dimethylarginine. $J$ Am Coll Cardiol 2005; 46:1693-1701.

22 Aicher A, Heeschen C, Mildner-Rihm C, et al. Essential role of endothelial nitric oxide synthase for mobilization of stem and progenitor cells. Nat Med 2003; 9:1370-1376.

23 Lee PC, Kibbe MR, Schuchert MJ, et al. Nitric oxide induces angiogenesis and upregulates alpha(v)beta(3) integrin expression on endothelial cells. Microvasc Res 2000; 60:269-280.

24 Sasaki K, Murohara T, Ikeda H, et al. Evidence for the importance of angiotensin II type 1 receptor in ischemia-induced angiogenesis. J Clin Invest 2002; 109:603-611.

25 Toko H, Zou Y, Minamino T, et al. Angiotensin II type 1a receptor is involved in cell infiltration, cytokine production, and neovascularization in infarcted myocardium. Arterioscler Thromb Vasc Biol 2004; 24:664-670.

26 Takahashi T, Kalka C, Masuda H, et al. Ischemia- and cytokineinduced mobilization of bone marrow-derived endothelial progenitor cells for neovascularization. Nat Med 1999; 5:434-438.

27 Verma S, Kuliszewski MA, Li SH, et al. C-reactive protein attenuates endothelial progenitor cell survival, differentiation, and function: further evidence of a mechanistic link between C-reactive protein and cardiovascular disease. Circulation 2004; 109:2058-2067.

28 Walter DH, Rittig K, Bahlmann FH, et al. Statin therapy accelerates reendothelialization: a novel effect involving mobilization and incorporation of bone marrow-derived endothelial progenitor cells. Circulation 2002; 105:3017-3024.

29 Tepper OM, Galiano RD, Capla JM, et al. Human endothelial progenitor cells from type II diabetics exhibit impaired proliferation, adhesion, and incorporation into vascular structures. Circulation 2002; 106:2781-2786.

30 Laufs U, Werner N, Link A, et al. Physical training increases endothelial progenitor cells, inhibits neointima formation, and enhances angiogenesis. Circulation 2004; 109:220-226.

31 Strehlow K, Werner N, Berweiler J, et al. Estrogen increases bone marrow-derived endothelial progenitor cell production and diminishes neointima formation. Circulation 2003; 107:30593065.

32 Yamaguchi J, Kusano KF, Masuo O, et al. Stromal cell-derived factor-1 effects on ex vivo expanded endothelial progenitor cell recruitment for ischemic neovascularization. Circulation 2003; 107:1322-1328. 